\title{
COMMENTARY
}

\section{Effects of immune checkpoint inhibitors on cancer patients with pre- existing autoimmune disease}

\author{
Fukumi Furukawa* \\ Department of Dermatology, Takatsuki Red Cross Hospital, Takatsuki City, Osaka 569-1096, Japan. E-mail: ffuruka- \\ wa@takatsuki.jrc.or.jp
}

Keywords: Immune Checkpoint Inhibitors; Malignant Melanoma; Cancer; Immune-related Adverse Events; Pre-existing Autoimmune Disease

\section{ARTICLE INFO}

Article history:

Received 12 April 2021

Received in revised form 11 May 2021

Accepted 17 May 2021

Available online 17 May 2021

\section{COPYRIGHT}

Copyright $(02021$ Fukumi Furukawa. doi: 10.24294/ti.v5.i1.1250

EnPress Publisher LLC. This work is licensed under the Creative Commons Attribution-NonCommercial 4.0 International License (CC BY-NC 4.0).

http://creativecommons.org/licenses/ by/4.0/
Since immune checkpoint inhibitors (ICIs) imbalance the immune system, this drug causes a secondary autoimmune condition ${ }^{[1-3]}$. Then on the contrary, it is a very interesting question whether they can be used in cancer patients with autoimmune diseases or what side effects they cause.

The Netherlands study group reported that response rates for ICIs in patients with advanced melanoma are comparable with or without pre-existing autoimmune disease (PAD) such as rheumatologic PAD, endocrine PAD, inflammatory bowel disease (IBD) PAD, or others ${ }^{[4]}$. Among patients with coexisting autoimmune diseases when receiving anti-CTLA-4 therapy, anti-PD-1 therapy, or a combination of both, the incidence of grade 3 or higher new toxicity profile called immune-related adverse events (irAEs) occurred in 30\%, 17\%, and 44\%, respectively. In patients with non-autoimmune diseases, it was 30\%, 13\% and $48 \%$, respectively. Compared to non-autoimmune diseases, patients with autoimmune diseases were more likely to discontinue anti-PD-1 therapy due to toxicity. Anti-PD-1-induced colitis was common in patients with IBD. Patients who received anti-CTLA-4, anti-PD-1, and combination therapy had similar objective response rates in autoimmune diseases and non-autoimmune diseases patients. So, taken together, the authors concluded that there was no difference in survival with or without autoimmune diseases.

Based on the meta-analysis of observational studies and nationwide multicenter cohort study ${ }^{[5,6]}$, immune toxicities are frequent in ICI-treated patients with PAD but often mild and manageable without discontinuing therapy. It was concluded that ICI treatments are also effective in PAD patients although immunosuppressive therapies at baseline are associated with poorer outcomes.

At present, ICI may be used in cancer patients with autoimmune diseases. It should be noted that many of the reported cases are rheumatoid arthritis (RA). Risks and benefits should be considered with special cares and attentions for multi-organ autoimmune diseases and/ or collagen diseases such as systemic lupus erythematosus, which has different mechanisms from RA. 


\section{Conflict of interest disclosure}

None declared.

\section{References}

1. Furukawa F. The Nobel Prize in Physiology or Medicine 2018 was awarded to cancer therapy by inhibition of negative immune regulation. Trends in Immunotherapy 2018; 2. doi:10.24294/ti.v2.i3.1065

2. Seidel JA, Otsuka A, Kabashima K. Treating tumors with immune checkpoint inhibitors: Rationale and limitations. Trends in Immunotherapy 2017; 1 (1): 2-9. doi: $10.24294 /$ ti.v1.i1.20

3. Furukawa F. Immune checkpoint inhibitors and irAEs. Trends in Immunotherapy 2018; 2. doi:10.242 94/ti.v2.i2.930
4. Van der Kooij MK, K Suijkerbuijk KPM, Aarts MJB, et al. Safety and efficacy of checkpoint inhibition in patients with melanoma and preexisting autoimmune disease: A cohort study. Annals of Internal Medicine 2021; 174(5): 641-648. doi: 10.732 6/M20-3419.

5. Xie W, Huang H, Xiao S, et al. Immune checkpoint inhibitors therapies in patients with cancer and preexisting autoimmune diseases: A meta-analysis of observational studies. Autoimmunity Reviews 2020; 19(12). doi: 10.1016/j.autrev.2020.102687.

6. Tison A, Quéré G, Misery L, et al. Safety and efficacy of immune checkpoint inhibitors in patients with cancer and preexisting autoimmune disease: A nationwide, multicenter cohort study. Arthritis Rheumatol 2019; 71(12): 2100-2111. doi: 10.1002/art. 41068. 